\title{
3 Research Square

\section{'Relearning How to Think': A Brief Online Intervention to Modify Biased Interpretations in Emotional Disorders. Study Protocol for a Randomised Controlled Trial}

Inés Nieto ( $\square$ inenieto@ucm.es )

Complutense University of Madrid: Universidad Complutense de Madrid https://orcid.org/0000-00018902-3791

\section{Carmelo Vazquez}

Complutense University of Madrid

Study protocol

Keywords: Cognitive Bias Modification, Ambiguous Interpretations, Emotional Disorders

Posted Date: March 10th, 2021

DOl: https://doi.org/10.21203/rs.3.rs-290225/v1

License: (c) (1) This work is licensed under a Creative Commons Attribution 4.0 International License. Read Full License

Version of Record: A version of this preprint was published at Trials on July 31st, 2021. See the published version at https://doi.org/10.1186/s13063-021-05459-3. 


\section{Abstract}

Background: Cognitive biases seem to play an important role in the development and maintenance of emotional disorders such as depression and anxiety. Novel procedures, known as Cognitive Bias Modification (CBM), aim to reduce these dysfunctional information processing modes. This study develops a brief online intervention program to modify biased interpretations in depression and anxiety, overcoming some methodological issues that have been addressed in previous literature.

Methods: Volunteer participants will be recruited via social media, posters and universities. They will be randomly assigned to an experimental group or a waiting list control group. Both groups will attend two assessment sessions at the lab. The assessments will consist of questionnaires measuring cognitive and emotional variables as well as three experimental tasks to measure cognitive biases (i.e., attention, memory and interpretation). After the first assessment session, only participants in the experimental group will receive a link to follow the four-sessions program at home. All participants will receive, via email, follow-up questionnaires two weeks and three months after the second assessment session.

Discussion: This study will test an online program potentially beneficial to modify cognitive variables in disorders such as depression and anxiety. Several limitations of previous CBM procedures are addressed and the impact of the program both on cognitive performance and clinical symptoms will be explored.

Trial registration: This trial was prospectively registered on June 17, 2019 with the ClinicalTrials.gov NCT03987477

\section{Background}

Anxiety and mood disorders are the most common mental disorders in the general population, showing an $18.1 \%$ and $9.5 \%$ 12-month prevalence, respectively [1]. Both disorders are associated with high social and economic costs, as well as high rates of chronicity and relapse $[2,3]$.

High patterns of comorbidity between anxiety and mood disorders are consistent across studies [1, 4]. This comorbidity could be due, at least in part, to the fact that these disorders share transdiagnostic factors such as rumination [5], and are also likely to share some general risk factors [6]. Cognitive theories point to dysfunctional thinking as one of the main variables related to the onset and maintenance of these emotional disorders [6-8] According to these influential theories, depressed and anxious individuals show specific cognitive dysfunctions that can ultimately lead to symptom development [9]-10]. Several types of dysfunctions have been distinguished in regard to the onset and maintenance of emotional disorders. A useful heuristic to clarify diverse types of cognitive activity intervening in psychopathology is the so-called "cognitive taxonomy", initially proposed by Ingram \& Kendall [11]. This taxonomy differentiates between structura/ variables (i.e., broad cognitive schemas through which information is filtered, represented and organised); operationa/variables (i.e., the mechanisms such as attentional, interpretation, or memory biases, by which cognitive structures work); product variables (i.e., the tangible outputs such as thoughts, images and memories, with which 
clinicians typically work with their clients). In general, cognitive biases have been defined as errors or distortions related to the operational domain that occur systematically at different times and across distinctive situations, reflecting 'irrational' modes of perceiving and elaborating information $[12,13]$.

\section{Cognitive Bias Modification (CBM)}

Cognitive Bias Modification (CBM) is a recent approach developed to alter cognitive biases and explore the link between them and symptoms development. CBM procedures have mainly been aimed at modifying attentional, memory, or interpretation biases. Besides their clinical utility, these procedures are theoretically ambitious as their rationale is that a causal link between cognition and emotion would be demonstrated if changes in cognition are accompanied by consequent changes in emotion. Thus, the efficacy of CBM would provide support to the etiological role of cognition in emotional disorders. Although it has been applied in different conditions, such as eating disorders, substance abuse or angerrelated problems [14], the main focus has been in anxiety and depression [15-18].

Mathews and Mackintosh [19], in their pioneer study in the field of CBM for interpretation processes (CBM-I), used a paradigm to induce negative interpretation biases in healthy individuals and found a possible causal link to anxiety. Since then, studies using different training paradigms have found an association between change in interpretation bias and symptoms. Recent meta-analyses show evidence of correlation between change in interpretation bias and negative mood [20], and significant differences between CBM training and control groups in anxiety and depression measures [17]. Some transdiagnostic factors, such as rumination, have also been found to improve with CBM-I [21]. A review of 12 metaanalyses of CBM procedures [18] revealed that there was a significant effect in 8 out of 10 meta-analyses for anxiety and 3 out of 7 meta-analyses for depression. Authors also found significant changes in attention biases in 8 out of 9 meta-analyses of studies using Attentional Bias Modification procedures $(\mathrm{ABM})$, and changes in interpretation biases in the 3 meta-analyses on CBM-I. All this evidence reflects a possible causal link between cognitive biases and symptoms that, at least in the case of interpretation biases, might suggest the existence of a causal connection with a depressed mood in particular [22].

Although results are promising, there are some limitations in the current CBM procedures [23, 24]. First of all, there are some variables that could affect the efficacy of CBM but remain unexplored. For example, although there are some studies indicating that the use of mental imagery during CBM procedures could have a beneficial impact on CBM [25-27], it is still unclear whether this factor is relevant [17, 20]. Also, most CBM procedures are based on the idea that repetitive exposure to a specific way of processing information leads to its automatic use later in daily life [28]. Yet, the theoretical support to this mechanistic and repetitive procedure (that typically involves hundreds or even thousands of trials) is still not clear. In fact, it could be possible that CBM procedures focused on enhancing elaborative rather than automatic processing modes could be more beneficial for disorders like depression where those elaborative mechanisms seem to be more affected than automatic ones [26]. 
Another methodological recommendation to improve CBM paradigms is the use of direct and indirect measures to evaluate cognitive change $[14,22]$. This involves asking participants to directly respond to a series of interpretations (e.g. plausibility ranking or the scrambled sentence task) together with nonconscious measures of interpretations (e.g. reaction time or jumping to conclusions measure). The evaluation of long term benefits, when participants may have faced possible stressors in real life, has also been encouraged by previous research [29]. Also, cognitive biases at different processing levels (attention, interpretation and memory) have traditionally been studied independently from each other but some authors now state the need to know how they interact with each other [30, 31].

\section{Objectives and hypotheses}

The purpose of the study is to design a brief online intervention aimed at reducing the interpretation of negative emotional cognitive biases. The intervention will be applied to an experimental group to analyse its impact on cognitive and emotional variables in comparison to a waiting-list control group. More specifically, the intervention is framed within the field of CBM-I but, instead of using repetitive training, it is based on the techniques frequently used in cognitive-behavioural therapies (e.g. $(31,32,33)$. Thus, while original CBM studies (e.g. [19]) were designed to train participants to change cognitive interpretation biases in an automatic manner, the current intervention aims to teach participants the meaning and consequences of emotional cognitive biases, plus how to modify them.

This study also aims to improve with regard to the procedures used in previous CBM procedures. First, based on previous evidence [35], mental imagery is used in order to shed some light on its role when modifying cognitive processes. Second, direct and indirect measures of cognitive performance are used to complement the information provided by self-report questionnaires. Moreover, these measures evaluate the three different domains proven to be affected by them (attention, interpretation and memory) [36], giving the need to explore their interplay [37]. Finally, given that it is not clear the maintenance of CBM effects, a longitudinal follow-up was used to explore the continuation of benefits in time and the dynamics of the different processing biases (attention, interpretation and memory).

It is hypothesised that there will be a significant change in interpretation bias (less negative or more positive/neutral) from pre-intervention to post-intervention in the experimental group in comparison to the control group. It is also expected that changes in interpretation biases will also be associated to significant changes in attention and memory biases (less negative or more positive/neutral) from preintervention to post-intervention in the experimental group in comparison to the control group. Finally, it is hypothesised that the intervention will help the experimental group to reduce symptoms of depression and anxiety and increase well-being from pre-intervention to post-intervention in comparison to the control group. Due to the lack of conclusive findings from previous studies, it is explored whether these changes are maintained over time (after 2 weeks and 3 months) and the temporal dynamics of the different cognitive bias processes (attention, interpretation and memory).

\section{Methods}




\section{Participants and recruitment}

Sample size was calculated based on the estimated effect size of the change in interpretation bias before and after a CBM-I intervention ( $\mathrm{d}=.43$ ) according to a recent meta-analysis on the field [20]. Following G*Power calculations [38], the minimum sample size (a set at 0.05 , power at 0.95 ) to find a difference in a repeated-measures multivariate analysis of variance with one within-subjects factor (two time points) and one between-subjects factor (two groups) was 73 participants. $20 \%$ more participants will be recruited due to expected attrition [39].

Participants will be volunteer students from the Complutense University of Madrid who will be recruited via social media channels associated to the university and posters at the Faculty of Psychology. They will be offered course academic credits in exchange for their participation. Exclusion criteria will include any form of visual and/or auditory disability that makes participants unable to follow online sessions and lack of internet access. No restrictions will be placed regarding concomitant treatment during the study, although this information will be monitored during the assessment sessions.

This study is a randomised superiority trial with two parallel groups and a 1:1 allocation ratio. Participants will be randomly allocated (using a macro in excel) to the experimental group (cognitive bias intervention) or a control waiting list group. This control group was chosen due to the novelty of the intervention, for which a waiting list is recommended to get a first impression of its effects [40]. The procedure has been approved by the university ethics committee (Ref. 2018/19 - 017) and has been registered (ClinicalTrials.gov NCT03987477). Moreover, it follows the recommendations for a clinical trial protocol [41].

Intervention: 'Relearning how to think' program

This is a brief online intervention designed to modify negative emotional interpretation biases. The intervention is composed of four different sessions in audiovisual format with psychoeducational content, open-answer questions and exercises to be completed by users. Different cognitive biases, such as jumping to conclusions, mental filter, overgeneralisation and negative attributions are targeted in each session following classical descriptions of biases [7, 42]-see Fig. 1. The organisation of the sessions content was based on the Cognitive Error Rating Scales (CERS) [43], a manual created for therapists to evaluate cognitive errors during clinical sessions, and the CBM-errors [44], a clinical strategy to promote more benign interpretations following Beck's theory [24].

Each session of 'Relearning how to think' is composed of four different parts (see Fig. 2). In part 1, participants receive information about specific interpretation biases and are given examples in video format. Some of these videos are daily life scenes where professional actors represent examples of cognitive biases (following clinical vignettes described elsewhere)[6]. During each video (before the resolution of the scene), and in order to increase active involvement, participants have to complete an open-ended question about what could happen in those ambiguous situations. In the second part, users are informed about the risks of using negative interpretations and it is followed by the explanation of the 
strategies to avoid them (part 3). These strategies are based on "the 4 questions technique" [45], widely used in clinical practice. This technique involves 4 steps to re-evaluate the negative interpretation of a given situation: finding evidence for the negative thoughts, uncovering the cognitive bias present in the situation, identifying the negative consequences and creating alternative ways of thinking. Finally, during part 4, participants have the opportunity to practice the strategies in an exercise composed of imagery training [46] followed by negative scenarios in audio format aimed to be reinterpreted. Figure 3 shows the steps of the exercise. It starts with an imaginary training [46] aimed to make scenarios more vivid to users. Participants are presented a screen saying 'Close your eyes. Imagine.' for 1 second followed by a black screen during which a negative scenario is played in audio format with a female voice (e.g. Your partner travels to work by car and normally arrives home promptly every day. Today you notice that they are over an hour late. Your first thought is that there must have been a crash). Audio scenarios are daily life situations where negative interpretations arise, and participants are asked to imagine themselves in those situations. A beep is played for users to open their eyes and start with the exercise questions. First, they have to rate their mood (sadness, happiness, anxiety and anger) on a 10-point VAS scale based on the most frequent emotions experienced in daily life $[47,48]$. Then, they are guided to apply the 4 questions technique to each scenario. Finally, users evaluate the degree to which they believe in the new alternative thoughts and emotions generated by the new scenario (sadness, happiness, anxiety and anger).

\section{Cognitive bias measures}

Ambiguous Scenarios Test for Depression-II. The AST-D-II [49] is a self-report measure of interpretation biases. It consists of 15 ambiguous scenarios which participants have to rate on a scale from - 5 (very unpleasant) to 5 (very pleasant). Participants are asked to imagine each scenario as vividly as possible and as if it was happening to them (e.g. As you enter the room, the commission welcomes you and begins with the oral examination. After just a few minutes you know intuitively how the examination will go). Two parallel versions are used in counterbalanced order at baseline, post-treatment and in the followups. Internal consistency has been shown to be good $(a=0.87)$ for the general scale and also for its two short versions $A(a=0.77)$ and $B(a=0.78)$.

Mouse-based (gaze) Contingent Attention Task (MCAT) [50]. A variant of the original Scrambled Sentence Test (SST) [51] is used to measure interpretation bias while monitoring attention towards emotional stimuli, based on the same principles as in the Eye-gaze Contingent Attention Training (ECAT) [52]. At the beginning of the task, participants have to click a fixation cross at the left side of the screen to elicit natural left-to-right reading patterns. Then, the reading screen is presented with a six-word emotional scrambled sentence (e.g., "am winner born loser a I") where each word is hidden with a blank mask. Participants are instructed to move the mouse cursor over each mask to read the hidden word and mentally form a grammatically correct sentence using five of them. They are given a time limit of 14 seconds per sentence. This procedure is used to objectively measure attention biases toward emotional words (negative or positive). Then, the answer screen is presented with the six words unmasked for 
participants to click the order of the sentence they had mentally formed. In this section, participants are given a time limit of 7 seconds. The resulting ratio of correctly negative unscrambled sentences and correctly emotional unscrambled sentences is considered to be the index of automatic negative interpretation bias. To maximise the appearance of biases, at the beginning of the task participants are presented a six-digit number for 5 seconds and told to keep that number in mind during the entire task as they will be asked to retrieve it at the end of the task.

SST-Memory task. As a measure of memory biases, participants are given 5 minutes to remember the sentences they constructed during the SST. Following Everaert et al.'s procedure [31], the ratio of correctly recalled negative/positive scrambled sentences is a measure of memory bias.

Computerised beads task [53]. The beads task is a measure of probabilistic reasoning which was initially designed to measure jumping to conclusion (JTC) bias in schizophrenic patients [54]. The adapted version used in this study has two parts. The first part consists of presenting two jars with beads of two different colours in different ratios (e.g. 60 orange/40 purple, and 60 purple/40 orange). Participants are told that the program selects one of the jars to take beads randomly out of it and then return them. The instruction is to decide which jar is being used, based on the number of beads of each colour. The second part follows the same procedure with the difference being that the beads are all in white but present two different ratios of positive and negative adjectives (60 positive/40 negative, and 60 negative/40 positive). The number of beads viewed before reaching a decision is an index of jumping to conclusion bias.

\section{Symptom measures}

Depression, Anxiety and Stress Scale-21. The DASS [55] is a 21-item self-report questionnaire measuring symptoms of depression, anxiety and stress. Each of the three subscales contains 7 items and provide a different score for each construct. This questionnaire has shown good reliability with the following a values for the Depression, Anxiety and Stress scales, respectively: 0.84, 0.70 and 0.82 [56].

Patient Health Questionnaire-9. The PHQ-9 [57] is a 9-item self-report questionnaire to assess any present episodes of depression according to the DSM-IV diagnostic criteria. Each item is rated in frequency on a four-point scale from 1 (Not at all) to 4 (Nearly every day). This questionnaire has shown good reliability with a Cronbach's a of 0.89 [58]. An adapted PHQ-9 will also be used to measure past episodes of depression. In this study, the standard diagnostic cutoff score of PHQ-9 $\geq 10$ [59] will be used to create groups based on present and past episodes of depression.

GAD-7 [60] is a 7-item self-report questionnaire to assess any present episodes of anxiety according to the DSM-IV diagnostic criteria. Each item is rated on a four-point scale from 0 (Not at all) and 3 (Nearly every day), with the final score being between 0-21. An adapted version of this questionnaire was also used to measure past episodes of anxiety. The cutoff score used in this study to consider present or past episodes of anxiety was GAD-7 $\geq 10$, following the severity scale: minimal ( $0-4)$, mild (5-9), moderate (10-14) and serious (14-20) [65].

\section{Other measures}


Pemberton Happiness Index. The PHI [61] is an 11-item self-report questionnaire measuring general, eudaimonic, hedonic and social well-being. It has shown to have a very good reliability $(\alpha=.92)$.

Dysfunctional Attitudes Scale. The DAS [62] is a scale of 40 sentences reflecting dysfunctional cognitive schemas. Participants have to rate each sentence from 0 (not applicable to me) to 3 (highly applicable to me). The sum of the scores is an index of stable dysfunctional attitudes (i.e., a measure of cognitive structures). The DAS has been found to be a predictor of major depression [63] and it has shown to have good reliability $a=.70[64]$.

Ruminative Responses Scale. The RRS [65] (Nolen-Hoeksema \& Morrow, 1991) is composed of two subscales measuring rumination cognitive style. For the present study, only the 5 -item brooding subscale will be used in order to measure the tendency to ruminate about negative events. The scale has shown good reliability $(a=.93)[66]$.

The scale for mood assessment-EVEA [67] is a measure of current mood that participants take immediately before and immediately after each of the sessions of the program. It is included to reflect some possible reactions to the cognitive training procedure. Participants have to rate, from 0 to 10 , their current level of anger, happiness, anxiety, depression and boredom. Scores of each subscale (4 items each) are summed up providing an index of emotional change during the session.

Credibility and expectancy questionnaire. The CEQ [68] is a 6-item measure used to assess the expectancy and rationale credibility of participants regarding the online program they are offered before they start it. It consists of two subscales that measure credibility based on cognition (what you think) and treatment expectancy based on affect (what you feel). Both subscales have shown to have good internal consistency ( $a=.86$ for credibility, $a=.90$ for expectancy).

The Working Alliance Inventory for Internet interventions (WAI-I) [69] is a self-report measure to assess alliance in internet interventions. In this study, only the 8-item subscale of task and goal agreement with program was used in order to measure the level of concordance of the program with participants' interests. This measure was used at the end of the program to know if participants were satisfied with the result. An example of an item is 'Through the online program I have become clearer about the things I need to do to help improve my situation'. Cronbach's a for this subscale has been shown to be good (a $=.84)$.

Stressful Events Questionnaire (SE) [70] is a self-report scale to measure stressful situations that happen to participants between the second assessment and the follow-up ( 2 weeks and 3 months). The scale includes positive and negative ratings of high impact events as well as daily events related to different contexts (social, emotional, academic/occupational and 'other').

\section{Procedure}

Figure 4 shows the schedule of enrolment, intervention and assessment following the recommendations for clinical trials [41]. The main investigator will run a macro in excel for the randomisation of 
participants to the experimental and control groups. This method assigns a sequence of numbers with a different codification for each group. In any case, participants will be aware of the codification meaning. Participants will receive the questionnaires for the first assessment the day before the first assessment session in the lab, this will be given along with the consent form and an information file about the online program. During this session, the main investigator will explain the rationale of the intervention 'Relearning how to think' and will provide participants with the instructions to complete the program. They will need to log into an online platform created for the purpose of this study and create their own account and password to access the material. Information will be coded with the participant's number and only the main researchers will have access to the data. This data will be monitored by the main investigator as adherence control. When the participants first access the platform, they will find the first session available and only 24 hours later they will be allowed to complete the second one. This intermission between sessions aims to increase participants learning and avoid cognitive overload and boredom.

After a briefing on the intervention, participants will sign the informed consent (explaining their right to discontinue the study at any moment and with no consequences) and complete some demographic information along with the three experimental tasks (MCAT, computerised beads task and SST recall task). At the end of this first assessment, participants will be notified again after approximately 10 days for a second assessment session and the experimental group will be receiving, via email, the link to start the intervention online. The control group will be given the opportunity to complete the intervention right after the second assessment. To improve adherence, both groups will receive a reminder for the second assessment session the day before the date. Finally, those participants who agree will be sent some selected questionnaires for the follow-ups (after 2 weeks and after 3 months). To increase adherence during the follow-ups, participants will be sent up to a maximum of three reminders to complete questionnaires. Both groups will have the opportunity to complete a 'feedback question' to give their opinion about the intervention. This question will be included in the post-assessment for the experimental group and at the follow-ups for the control group.

\section{Analytic plan}

Demographic data and pre-treatment measures will be analysed to test for group differences with analysis of variance and chi squared test for nominal variables.

Complete case analyses will be conducted for those participants who complete all 4 online sessions and attend pre- and post-assessment evaluation sessions. A series of 2 (group: Experimental, Control) $\times 3$ (symptom group: never, present, past) $\times 2$ (time: pre-training, post-training) analyses of variance will be performed to evaluate the change between groups. The symptom group will be created based on present and past symptoms of depression and/or anxiety to explore their influence in the results. Intention-to-treat (ITT) analyses will be conducted with all participants, regardless of session or outcome measure completion. ITT mixed models (restricted maximum likelihood (REML) estimation) will be used to account for missing data [71]. Binary logistic regression will be used to evaluate the assumption that data is Missing at Random (MAR). Exploratory analyses will be conducted to study the interplay between the different cognitive bias scores. Finally, follow-up assessments will include a series of analyses of 
variance to evaluate group differences in time. All analyses will be performed in SPSS Statistics 20 with an a level of 0.05 .

\section{Discussion}

The current study will test the efficacy of a brief online intervention to target emotional negative cognitive biases. Although traditional CBM interventions are designed to change this type of dysfunctional processing in an automatic way [17], the rationale of 'Relearning how to think' is to increase participants' awareness of their own thought processes and guide them to change these processes, in a more effortful way, by following a clinically-oriented working frame.

The study uses a transdiagnostic conceptualisation of the role of cognitive biases in psychopathology. Given the high comorbidity between anxiety and mood disorders [1], having intervention tools that can tap both problems could be clinically useful. Some of the video scenarios designed for the program were based on examples proposed by previous transdiagnostic approaches [6] and the content is suitable for different common psychopathologies in which cognitive biases may play a role.

The study also addresses some of the questions that remain unanswered regarding CBM efficacy. It combines subjective (e.g. AST-D) along with objective measures (e.g. JTC measure, MCT) to assess cognitive biases. The present study aimed to complement both self-report and behavioural measures to avoid potential biases of respondents. Mental imagery is also used following the proposal that it may enhance CBM-I performance [14]. Holmes, et al. [46] found that mental imagery of emotional content has a beneficial impact on cognitive change. 'Relearning how to think' includes imagery training with the aim to potentiate vividness of the scenarios and promote interpretation change.

Furthermore, it explores in an innovative manner whether changes in interpretation biases may be associated to concomitant changes in attention and memory. There is very scarce basic research on the interrelation between different types of biases in emotional disorders [31] and this study will offer a unique opportunity to explore whether a specific intervention designed to change interpretation of ambiguous scenarios may also affect other domains of information processing.

Finally, there is an increasing interest in the use of online interventions and many researchers wonder if this format is also beneficial for individuals with clinical problems. The extant evidence suggests that psychological treatments delivered online can be as effective as face-to-face therapies $[72,73]$ and seem to overcome some of the limitations traditional therapies present [74]. For example, online sessions can be taken by the individual at any time, there is no need to wait to schedule dates, stigma is reduced and individuals increase their self-efficacy [75]. Specifically, CBM interventions seem to be highly suitable for the online format due to its flexibility in application, or the minimal requirement of supervision, in comparison to traditional therapies [76]. CBM could even be used in a self-management way, so that it could be applied to patients waiting for treatment or presenting vulnerability factors [28]. In sum, we expect that this study will offer new responses to some of the challenges CBM procedures face to make 
them more feasible, efficient and more capable of providing answers to some theoretical issues related to the complex relations between emotion, cognition and clinical psychology.

\section{Abbreviations}

ABM: Attentional Bias Modification; AST-D-II: Ambiguous Scenarios Test for Depression-II; CBM: Cognitive Bias Modification; CBM-I: Cognitive Bias Modification of Interpretations; CEQ: Credibility and Expectancy Questionnaire; DAS: Dysfunctional Attitudes Scale; DASS-21: Depression, Anxiety and Stress Scale-21; ECAT: Eye-gaze Contingent Attention Training; EVEA: Scale for mood; GAD-7: Generalised Anxiety Disorder Scale-7; ITT: Intention-To-Treat; JTC: Jumping To Conclusions; MANOVA: Multivariate Analysis Of Variance; MAR: Missing At Random; MCAT: Mouse-based (gaze) Contingent Attention Task; PHI: Pemberton Happiness Index; PHQ-9: Patient Health Questionnaire-9; REML: Restricted Maximum Likelihood; RRS: Ruminative Responses Scale; SE: Stressful Events Questionnaire; SST: Scrambled Sentence Test; WAI-I: The Working Alliance Inventory for Internet interventions.

\section{Declarations}

\section{Trial status}

The trial was registered on 17th June 2019, with ClinicalTrial.gov NCT03987477. The study started recruiting participants in September 30th 2019 recruiting participants and it is estimated to be completed by December 30th 2020.

https://clinicaltrials.gov/ct2/show/NCT03987477?term=vazquez+and+nieto\&draw=2\&rank=1

\section{Ethics approval and consent to participate}

This project has been approved by the School of Psychology Ethics Committee on June 17, 2019 (Ref. 2018/2019-017). All participants will provide informed consent before starting the first study session. To ensure anonymity, we will replace the names of participants with code numbers to process and analyse the data.

\section{Consent for publication}

All participants are asked for their consent to report on the results in publications.

\section{Availability of data and materials}

Not applicable

\section{Acknowledgements}

We thank Irene-López for her initial thoughts on the conceptualisation of the study and Marta Miragall, Simon Blackwell, Alvaro Sánchez and Vanesa Peinado for their valuable contributions to the contents of 
the study. We also thank Víctor García and Alvaro Franco for their technical support in implementing the online program, Elena Robles and Eva Moreno for their help in several stages of the study, Jamie O'Grady for his help in editing the paper, and all the students who voluntarily took part in the pilot study of the program (Ada Arteaga, Elena Castello, Andrea Cuesta, Beatriz Estefanía, and Juan José Rodrigo Apio).

\section{Funding}

This work was supported by a predoctoral fellowship CT17/17-CT18/17 UCM to IN. This funding source had no role in the design of this study and will not have any role during its execution, analyses, interpretation of the data, or decision to submit results.

\section{Authors' contributions}

IN and CV developed the study concept and study design. Testing and data collection will be performed by IN. Data analysis and interpretation will be performed by IN under the supervision of CV. IN drafted the manuscript and was corrected and expanded by CV. Both authors approved the final version of the manuscript for submission.

Any modifications to the protocol which may impact the potential benefit of the patient or may affect patient safety will be notified to the university ethics committee Administrative changes and minor corrections will be agreed by both authors.

\section{Competing interest}

The authors declare that they have no competing interest.

\section{Authors' details}

Carmelo Vázquez, Faculty of Psychology, Complutense University of Madrid, 28223 Madrid (Spain) cvazquez@psi.ucm.es

Inés Nieto, inenieto@ucm.es, Faculty of Psychology, Complutense University of Madrid, 28223 Madrid (Spain)

\section{References}

1. Kessler RC, Chiu WT, Demler O, Walters EE. Prevalence, severity, and comorbidity of 12-month DSM-IV disorders in the National Comorbidity Survey Replication. Arch Gen Psychiatryeneral psychiatry. 2005;62:617-27.

2. Whiteford HA, Degenhardt L, Rehm J, Baxter A, Ferrari AJ, Erskine HE, Vos T. Global burden of disease attributable to mental and substance use disorders: Findings from the Global Burden of Disease Study 2010. Lancet. 2013;382:1575-86. 
3. Collins PY, Patel V, Joestl SS, March D, Insel TR, Daar AS, et al. Grand challenges in global mental health. Nature. 2011;475:27-30.

4. Wittchen H-U. Generalized anxiety disorder: Prevalence, burden, and cost to society. Depress Anxiety. 2002;16:162-71.

5. McLaughlin KA, Nolen-Hoeksema S. Rumination as a transdiagnostic factor in depression and anxiety. Behav Res Ther. 2011;49:186-93.

6. Barlow, et al. Unified protocol for transdiagnostic treatment of emotional disorders: Therapist guide. New York: Oxford University Press; 2011.

7. Beck AT. Cognitive therapy and the emotional disorders. New York: Meridian; 1976.

8. Beck AT. Depression: Clinical, experimental, and theoretical aspects. Pennsylvania: University of Pennsylvania Press; 1967.

9. Mineka S, Watson D, Clark LA. Comorbidity of anxiety and unipolar mood disorders. Annu Rev Psychol. 1998;49:377-412.

10. Nieto I, Robles E, Vazquez C. Self-reported cognitive biases in depression: A Meta-analysis. 2020.

11. Ingram RE, Kendall PC. Cognitive clinical psychology: Implications of an information processing perspective. In: Ingram RE, editor. Inf Process approaches to Clin Psychol. Orlando: Academic Press; 1986. pp. 3-21.

12. Haselton MG, Nettle D, Andrews. and PW. The evolution of cognitive bias. In: Buss DM, editor. Handb Evol Psychol. Hoboken: John Wiley \& Sons Inc.; 2005. pp. 724-46.

13. Kahneman D. Thinking, fast and slow. Barcelona: Penguin Random House Editorial Group; 2012.

14. MacLeod C, Koster EHW, Fox E. Whither cognitive bias modification research? Commentary on the special section articles. J Abnorm Psychol. 2009;118:89-99.

15. Hakamata Y, Lissek S, Bar-Haim Y, Britton JC, Fox NA, Leibenluft E, et al. Attention Bias Modification treatment: A meta-analysis toward the establishment of novel treatment for anxiety. Biol Psychiatry. 2010;68:982-90.

16. Hallion LS, Ruscio AM. A meta-analysis of the effect of cognitive bias modification on anxiety and depression. Psychol Bull. 2011;137:940-58.

17. Cristea IA, Kok RN, Cuijpers P. Efficacy of cognitive bias modification interventions in anxiety and depression: Meta-analysis. Br J Psychiatry. 2015;206:7-16.

18. Jones EB, Sharpe L. Cognitive bias modification: A review of meta-analyses. J Affect Disord. 2017;223:175-83.

19. Mathews A, Mackintosh B. Induced emotional interpretation bias and anxiety. J Abnorm Psychol. 2000;109:602-15.

20. Menne-Lothmann C, Viechtbauer W, Höhn P, Kasanova Z, Haller SP, Drukker M, et al How to boost positive interpretations? A meta-analysis of the effectiveness of cognitive bias modification for interpretation. Voracek M, editor. PLoS One. 2014;9:e100925. 
21. Hertel P, Mor N, Ferrari C, Hunt O, Agrawal N. Looking on the dark side: Rumination and cognitive-bias modification. Clin Psychol Sci. 2014;2:714-26.

22. Everaert J, Podina IR, Koster EH. A comprehensive meta-analysis of interpretation biases in depression. Clin Psychol Rev. 2017;58:33-48.

23. Koster EHW, Bernstein A. Introduction to the special issue on Cognitive bias modification: Taking a step back to move forward? J Behav Ther Exp Psychiatry. 2015;49:1-4.

24. Lee J-S, Mathews A, Shergill S, Yiu Chan DK, Majeed N, Yiend J. How can we enhance cognitive bias modification techniques? The effects of prospective cognition. J Behav Ther Exp Psychiatry. 2015;49:120-7.

25. Holmes EA, Lang TJ, Moulds ML, Steele AM. Prospective and positive mental imagery deficits in dysphoria. Behav Res Ther. 2008;46:976-81.

26. Duque A, López-Gómez I, Blanco I, Vázquez C. Modificación de Sesgos Cognitivos (MSC) en depresión: Una revisión crítica de nuevos procedimientos para el cambio de sesgos cognitivos. Ter psicológica. 2015;33:103-16.

27. Hitchcock C, Werner-Seidler A, Blackwell SE, Dalgleish T. Autobiographical episodic memory-based training for the treatment of mood, anxiety and stress-related disorders: A systematic review and meta-analysis. Clin Psychol Rev. 2017;52:92-107.

28. Schartau PES, Dalgleish T, Dunn BD. Seeing the bigger picture: Training in perspective broadening reduces self-reported affect and psychophysiological response to distressing films and autobiographical memories. J Abnorm Psychol. 2009;118:15-27.

29. Vazquez C, Blanco I, Sanchez A, McNally RJ. Attentional bias modification in depression through gaze contingencies and regulatory control using a new eye-tracking intervention paradigm: Study protocol for a placebo-controlled trial. BMC Psychiatry. 2016;16:439.

30. Sanchez A, Duque A, Romero N, Vazquez C. Disentangling the interplay among cognitive biases: Evidence of combined effects of attention, interpretation and autobiographical memory in depression. Cognit Ther Res. 2017;41:829-41.

31. Everaert J, Duyck W, Koster EH. Attention, interpretation, and memory biases in subclinical depression: A proof-of-principle test of the combined cognitive biases hypothesis. Emotion. 2014;14:331-40.

32. Beck AT, Rush AJ, Shaw BF, Emery G. Cognitive therapy of depression. New York: Guildford; 1979.

33. Greenberger D, Padesky CA. Mind over mood: Change how you feel by changing the way you think. 2nd ed. New York: Guilford Press; 2015.

34. Leahy RL. Cognitive therapy techniques: A practitioner's guide. 2nd ed. New York: Guilford Publications; 2017.

35. Lang TJ, Blackwell SE, Harmer CJ, Davison P, Holmes EA. Cognitive Bias Modification using mental imagery for depression: Developing a novel computerized intervention to change negative thinking styles. Eur J Pers. 2012;26:145-57. 
36. Gotlib IH, Joormann J. Cognition and depression: Current status and future directions. Annu Rev Clin Psychol. 2010;6:285-312.

37. Everaert J, Koster EHW, Derakshan N. The combined cognitive bias hypothesis in depression. Clin Psychol Rev. 2012;32:413-24.

38. Faul F, Erdfelder E, Buchner A, Lang A-G. Statistical power analyses using G*Power 3.1: Tests for correlation and regression analyses. Behav Res Methods. 2009;41:1149-60.

39. Crutzen R, Viechtbauer W, Spigt M, Kotz D. Differential attrition in health behaviour change trials: A systematic review and meta-analysis. Psychol Health. 2015;30:122-34.

40. Gold SM, Enck P, Hasselmann H, Friede T, Hegerl U, Mohr DC, et al. Control conditions for randomised trials of behavioural interventions in psychiatry: A decision framework. The Lancet Psychiatry. 2017;4:725-32.

41. Chan A-W, Tetzlaff JM, Gotzsche PC, Altman DG, Mann H, Berlin JA, et al. SPIRIT 2013 explanation and elaboration: Guidance for protocols of clinical trials. BMJ. 2013;346:e7586.

42. Burns DD, Beck AT. Feeling good: The new mood therapy. New York: Avon; 1999.

43. Drapeau M, Perry JC, Dunkley D, Blake E. Cognitive Errors Rating Scales. Canada: McTavish; 2010.

44. Lester KJ, Mathews A, Davison PS, Burgess JL, Yiend J. Modifying cognitive errors promotes cognitive well being: A new approach to bias modification. J Behav Ther Exp Psychiatry. 2011;42:298-308.

45. Beck AT, Emery G, Greenberg R. Anxiety and phobias: A cognitive approach. New York: Basic Books; 1985.

46. Holmes EA, Lang TJ, Shah DM. Developing interpretation bias modification as a 'cognitive vaccine' for depressed mood: Imagining positive events makes you feel better than thinking about them verbally. J Abnorm Psychol. 2009;118:76-88.

47. Trampe D, Quoidbach J, Taquet M. Emotions in everyday life. PLoS One. 2015;10:e0145450.

48. Cowen AS, Keltner D. Self-report captures 27 distinct categories of emotion bridged by continuous gradients. Proc Natl Acad Sci. 2017;114:E7900-9.

49. Rohrbacher $\mathrm{H}$, Reinecke $A$. Measuring change in depression-related interpretation bias: Development and validation of a parallel ambiguous scenarios test. Cogn Behav Ther. 2014;43:239-50.

50. Sanchez-Lopez A, De Raedt R, van Put J, Koster EHW. A novel process-based approach to improve resilience: Effects of computerized mouse-based (gaze)contingent attention training (MCAT) on reappraisal and rumination. Behav Res Ther. 2019;118:110-20.

51. Wenzlaff RM, Bates DE. Unmasking a cognitive vulnerability to depression:How lapses in mental control reveal depressive thinking. J Pers Soc Psychol. 1998;75:1559-71.

52. Sanchez A, Everaert J, Koster EHW. Attention training through gaze-contingent feedback: Effects on reappraisal and negative emotions. Emotion. 2016;16:1074-85.

53. Huq SF, Garety PA, Hemsley DR. Probabilistic judgements in deluded and non-deluded subjects. Q J Exp Psychol Sect A. 1988;40:801-12. 
54. Garety PA, Freeman D, Jolley S, Dunn G, Bebbington PE, Fowler DG, et al. Reasoning, emotions, and delusional conviction in psychosis. J Abnorm Psychol. 2005;114:373-84.

55. Lovibond PF, Lovibond SH. The structure of negative emotional states: Comparison of the Depression Anxiety Stress Scales (DASS) with the Beck Depression and Anxiety Inventories. Behav Res Ther. 1995;33:335-43.

56. Bados A, Solanas A, Andrés R. Psychometric properties of the Spanish version of Depression, Anxiety and Stress Scales (DASS). Psicothema. 2005;17:679-83.

57. Spitzer RL, Kroenke K, Williams JB. PHQPCSG. Validation and utility of a self-report version of PRIME-MD: The PHQ primary care study. JAMA. 1999;282:1737-44.

58. Kroenke K, Spitzer RL, Williams JBW. The PHQ-9: Validity of a brief depression severity measure. J Gen Intern Med. 2001;16:606-13.

59. He C, Riehm E, Saadat N, Levis W, Ziegelstein RC, Akena H, et al. The Accuracy of the Patient Health Questionnaire-9 Algorithm for Screening to Detect Major Depression: An Individual Participant Data Meta-Analysis. Psychother Psychosom. 2020;89:25-37.

60. Spitzer RL, Kroenke K, Williams JBW, Löwe B. A brief measure for assessing generalized anxiety disorder. JAMA Intern Med. 2006;166:1092.1097.

61. Hervas G, Vazquez C. Construction and validation of a measure of integrative well-being in seven languages: The Pemberton Happiness Index. Health Qual Life Outcomes. 2013;11:66.

62. Weissman A, Beck A. The Dysfunctional Attitude Scale: A preliminary investigation. Pap Present Annu Meet Am Educ Res Assoc. 1978. p. 1-33.

63. Rude SS, Durham-Fowler JA, Baum ES, Rooney SB, Maestas KL. Self-report and cognitive processing measures of depressive thinking predict subsequent major depressive disorder. Cognit Ther Res. 2010;34:107-15.

64. Sanz J, Vázquez C. Adaptación española de la Escala de Actitudes Disfuncionales (DAS) de Beck: propiedades psicométricas y clínicas. Análisis y Modif Conduct. 1993;67:705-50.

65. Nolen-Hoeksema S, Morrow J. A prospective study of depression and posttraumatic stress symptoms after a natural disaster: The 1989 Loma Prieta earthquake. J Pers Soc Psychol. 1991;61:115-21.

66. Hervás Torres G. Adaptación al castellano de un instrumento para evaluar el estilo rumiativo. Rev Psicopatología y Psicol Clínica. 2008;13.

67. Sanz J. Un instrumento para evaluar la eficacia de los procedimientos de inducción de estado de ánimo: La "Escala de Valoración del Estado de Ánimo" (EVEA). Análisis y Modif Conduct. 2001;27:71-110.

68. Devilly GJ, Borkovec TD. Psychometric properties of the credibility/expectancy questionnaire. J Behav Ther Exp Psychiatry. 2000;31:73-86.

69. Gómez Penedo JM, Berger T, grosse Holtforth M, Krieger T, Schröder J, Hohagen F, et al. The Working Alliance Inventory for guided Internet interventions (WAI-I). J Clin Psychol. 2019;76:973-86. 
70. Hernangomez L. Vulnerabilidad cognitiva a la depresión: Relación entre sesgos atencionales, autorepresentación y síntomas depresivos. Universidad Complutense de Madrid; 2012.

71. Salim A, Mackinnon A, Christensen H, Griffiths K. Comparison of data analysis strategies for intentto-treat analysis in pre-test-post-test designs with substantial dropout rates. Psychiatry Res. 2008;160:335-45.

72. Cuijpers P, Smit F, Oostenbrink J, de Graaf R, ten Have M, Beekman A. Economic costs of minor depression: A population-based study. Acta Psychiatr Scand. 2007;115:229-36.

73. Holmes EA, Ghaderi A, Harmer CJ, Ramchandani PG, Cuijpers P, Morrison AP, et al. The Lancet Psychiatry Commission on psychological treatments research in tomorrow's science. The Lancet Psychiatry. 2018;5:237-86.

74. Kazdin AE, Blase SL. Rebooting psychotherapy research and practice to reduce the burden of mental illness. Perspect Psychol Sci. 2011;6:21-37.

75. Andersson G. Internet-Delivered Psychological Treatments. Annu Rev Clin Psychol. 2016;12:157-79.

76. Yiend J, Lee J-S, Tekes S, Atkins L, Mathews A, Vrinten M, et al. Modifying interpretation in a clinically depressed sample using 'Cognitive Bias Modification-Errors': A double blind randomised controlled trial. Cognit Ther Res. 2014;38:146-59.

\section{Figures}

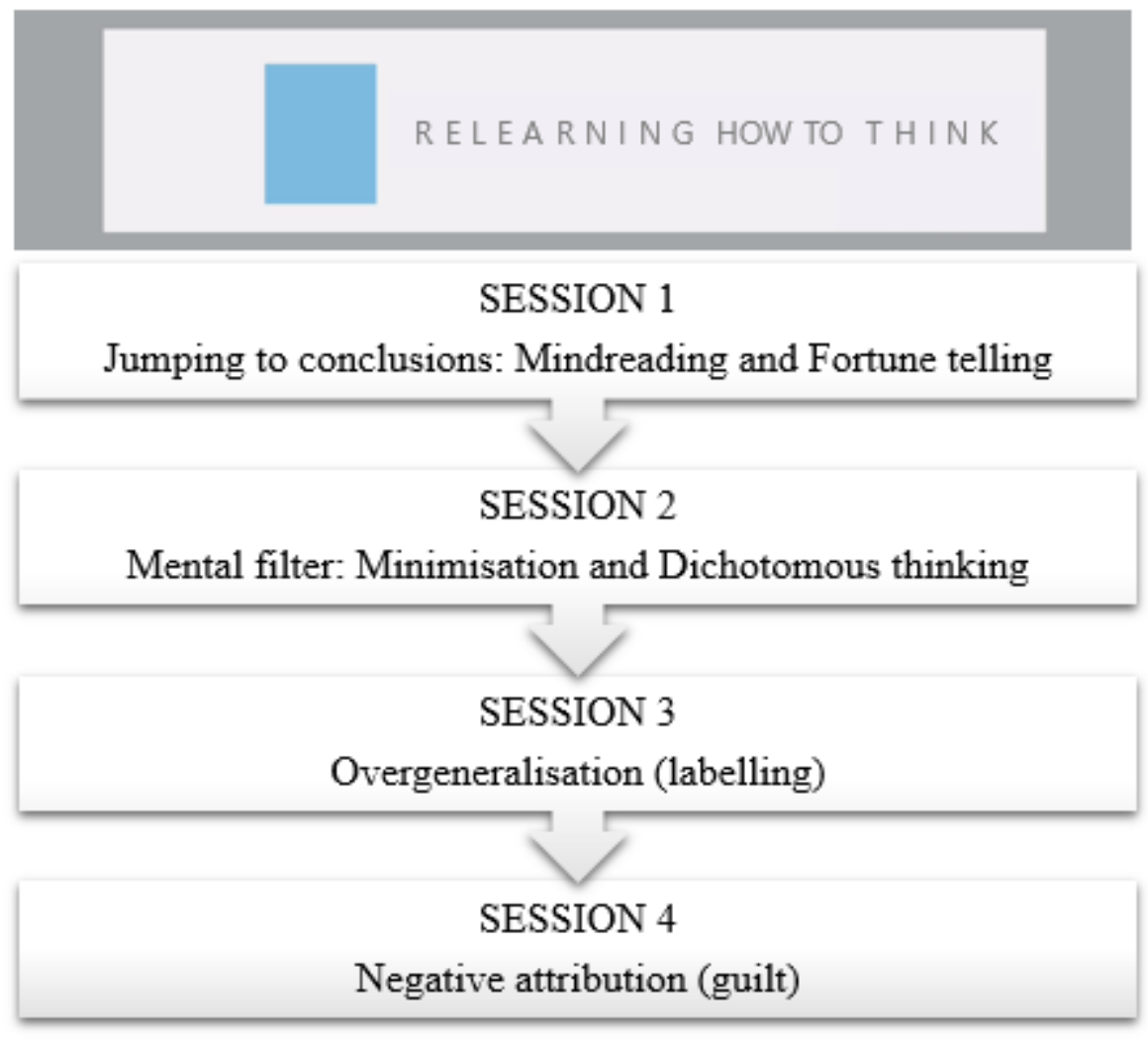

Figure 1 
Classification of the specific cognitive biases targeted in the four online sessions of the program.

RELEARNING HOW TO THINK

PART 1 Definition of bias and video example (open-questions)

PART 2 Risk of cognitive biases

PART 34 questions technique

\begin{tabular}{|c|c|c|c|}
\hline $\begin{array}{l}\text { 1. What is the } \\
\text { evidence? }\end{array}$ & $\begin{array}{l}\text { 2. Which cognitive } \\
\text { bias is present? }\end{array}$ & $\begin{array}{l}\text { 3. What are the } \\
\text { consequences? }\end{array}$ & $\begin{array}{c}\text { 4. Which are the } \\
\text { alternatives? }\end{array}$ \\
\hline \multicolumn{4}{|c|}{ PART 4 Exercise } \\
\hline Imagery training & \multicolumn{2}{|c|}{ Negative scenarios } & The 4 questions \\
\hline
\end{tabular}

Figure 2

Structure of each session of the program.

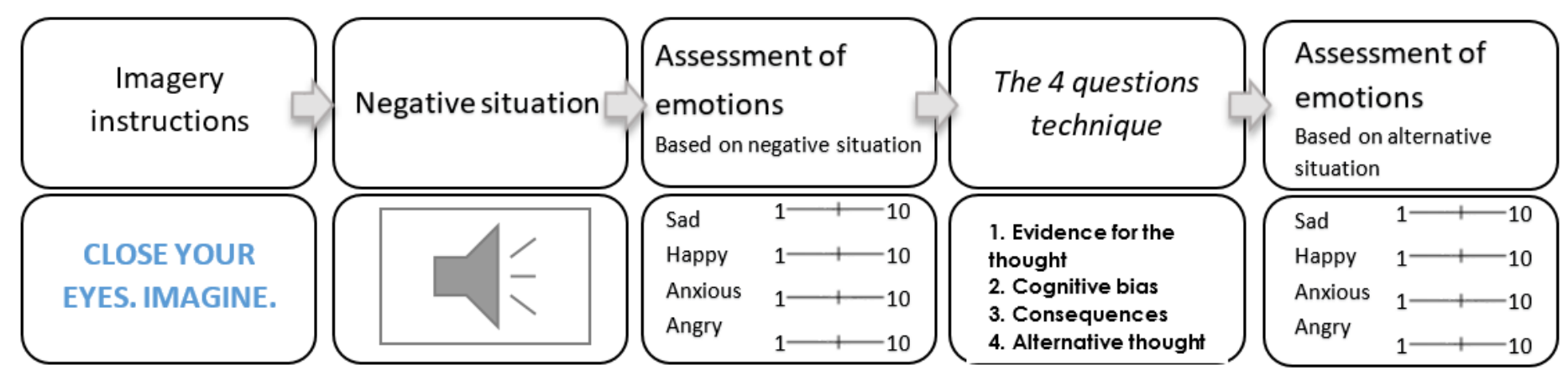

Figure 3 
Practice exercise of each session of the program.

\begin{tabular}{|c|c|c|c|c|c|c|}
\hline & \multicolumn{6}{|c|}{ STUDY PERIOD } \\
\hline & \multirow{2}{*}{$\begin{array}{c}\text { Eurolment } \\
-t_{s}\end{array}$} & \multirow{2}{*}{$\begin{array}{c}\text { Allocation } \\
0 \text { (Pre) }\end{array}$} & \multicolumn{3}{|c|}{ Post-allocation } & \multirow{2}{*}{$\begin{array}{c}\text { Close-out } \\
\begin{array}{c}\text { Follow-19y } \\
(3 m y)\end{array}\end{array}$} \\
\hline TIME POINT & & & $\begin{array}{c}10 \\
\text { dlays }\end{array}$ & Post & Follow-1p (2w) & \\
\hline \multirow{3}{*}{$\begin{array}{r}\text { ENROLMINT: } \\
\text { Informed consent } \\
\text { Allocation }\end{array}$} & & & & & & \\
\hline & $\mathrm{x}$ & & & & & \\
\hline & $\mathrm{x}$ & & & & & \\
\hline \multicolumn{7}{|l|}{$\begin{array}{r}\text { INTERVENTION } \\
\mathrm{S}:\end{array}$} \\
\hline $\begin{array}{r}\text { Online program } \\
\text { for the } \\
\text { experimental group }\end{array}$ & & & & & & \\
\hline \multicolumn{7}{|l|}{$\begin{array}{l}\text { Waining tome for } \\
\text { the control group }\end{array}$} \\
\hline \multicolumn{7}{|l|}{ ASSESSMENTS: } \\
\hline $\begin{array}{r}\text { Ambiguous } \\
\text { Scenarios Test for } \\
\text { Depression-II } \\
\text { (AST-D-II) }\end{array}$ & & $\mathrm{x}$ & & $\mathrm{x}$ & $\mathrm{x}$ & $\mathrm{x}$ \\
\hline $\begin{array}{r}\text { Depression, Anxiety } \\
\text { and Stress Scale-21 } \\
\text { (DASS-21) }\end{array}$ & & $\mathrm{x}$ & & $\mathrm{x}$ & $\mathrm{x}$ & $\mathrm{x}$ \\
\hline $\begin{array}{r}\text { Pemberton } \\
\text { Happiness Index } \\
\text { (PHI) }\end{array}$ & & $\mathrm{x}$ & & $\mathrm{x}$ & $\mathrm{x}$ & $\mathrm{x}$ \\
\hline $\begin{array}{r}\text { Mouse-bared (gare) } \\
\text { Contingent } \\
\text { Attention Task } \\
\text { (MCAT) }\end{array}$ & & $\mathrm{x}$ & & $\mathrm{x}$ & & \\
\hline
\end{tabular}

\begin{tabular}{|c|c|c|c|c|c|}
\hline \begin{tabular}{|r|} 
Scrambled Sentersce \\
Task-Memory (SST- \\
MU)
\end{tabular} & $\mathrm{x}$ & & $\mathrm{x}$ & & \\
\hline $\begin{array}{r}\text { Computerised } \\
\text { beadis task }\end{array}$ & $\mathrm{x}$ & & $\mathrm{x}$ & & \\
\hline $\begin{array}{r}\text { Dyaflartional } \\
\text { Attituder Scaie } \\
\text { (DASY) }\end{array}$ & $\mathrm{x}$ & & $\mathrm{x}$ & & \\
\hline $\begin{array}{r}\text { Ruminative } \\
\text { Responses Scaie } \\
\text { (RRS) }\end{array}$ & $\mathrm{x}$ & & $\mathrm{x}$ & & \\
\hline $\begin{array}{r}\text { Patient Heaith } \\
\text { Questionazive-9 } \\
\text { (PHO-9) }\end{array}$ & $\mathrm{x}$ & & & & \\
\hline \begin{tabular}{|r|} 
Generalised \\
Avxiety Disorder-7 \\
(GAD-7)
\end{tabular} & $\mathrm{x}$ & & & & \\
\hline \begin{tabular}{r|} 
The scaie for mood \\
assessment-EVEA
\end{tabular} & & $\mathrm{x}$ & & & \\
\hline $\begin{array}{r}\text { Credibility and } \\
\text { expectancy } \\
\text { questionnaire }\end{array}$ & $\mathrm{x}$ & & & & \\
\hline $\begin{array}{r}\text { The Working } \\
\text { Alliance Inventory } \\
\text { for Internet } \\
\text { ivterventions (WAI- } \\
\text { I) }\end{array}$ & & & $\mathrm{x}$ & & \\
\hline $\begin{array}{l}\text { Stressful Events } \\
\text { Questionaaire }\end{array}$ & & & & $\mathrm{x}$ & $\mathrm{x}$ \\
\hline
\end{tabular}

\section{Figure 4}

Schedule of enrolment, interventions and assessments.

\section{Supplementary Files}


This is a list of supplementary files associated with this preprint. Click to download.

- SPIRITCHECKLIST.doc 